\title{
Abgangsursachen und ihr Einfluss auf die Lebensleistung bei Sauen der Rassen Deutsches Edelschwein, Deutsche Landrasse und Pietrain
}

\begin{abstract}
Title of the paper: Culling reasons and their effect on lifetime productivity in sows of the breeds German Large White, German Landrace and Pietrain

Culling reasons in sows were analysed with respect to their importance for the different times of removal and their effects on longevity, length of productive life, number of piglets born alive and number of weaned piglets in the whole life of a sow. The investigation was based on records of 655 Large White (DE) sows, 13,005 German Landrace (DL) sows and 6,473 Pietrain (PI) sows born between1989 and 1994 and culled until 2002. In all the three breeds investigated, removal was highest due to fertility with $15 \%$ (DE and PI) and $20 \%$ (DL) and next highest due to diseases with 11 to $13 \%$. In PI sows, culling for low fattening performance and low efficiency in carcass yield ranked third (8 \%), while removal for old age (9 and $10 \%$, respectively) was more important in DE and DL sows. The main timespan for the different culling reasons were after the fourth farrowing due to infertility, between the 2nd and 5th farrowing due to low fattening performance and low efficiency in carcass yield, after the first farrowing due to reproduction problems and up to 4th farrowing due to diseases, while culling due to old age did not occur before the 5th farrowing. Heritabilities of reasons for removal were in the range of $h^{2}=0.04-0.08$ due to poor fertility, $h^{2}=0.01-0.09$ due to low fattening performance and low efficiency in carcass yield, $h^{2}=0.02$ due to reproductive problems, between $h^{2}=0.01$ and $\mathrm{h}^{2}=0.05$ due to diseases and between $\mathrm{h}^{2}=0.05$ and $\mathrm{h}^{2}=0.16$ due to miscellaneous reasons.
\end{abstract}

Key Words: sow, reasons for culling, lifetime performance, heritability

\section{Zusammenfassung}

Auf der Grundlage von 655 DE-Sauen, 13.005 DL-Sauen und 6.473 PI-Sauen, die zwischen 1989 und 1994 geboren wurden und im Jahr 2002 bereits abgegangen waren, wurden die Abgangsursachen hinsichtlich ihrer Bedeutung zu den unterschiedlichen Abgangszeiten der Sauen und ihres Einflusses auf die Lebensleistungsmerkmale Lebens- und Nutzungsdauer sowie der Anzahl insgesamt lebend geborener bzw. aufgezogener Ferkel untersucht. Bei allen drei Rassen hatten die Fruchtbarkeit mit 15 \% (DE und PI) und 20 \% (DL) und die Abgangsursache Krankheit mit 11 bis 13 \% den größten Einfluss auf den Abgang der Sauen. Bei den PI-Tieren folgte an dritter Stelle die Merzung der Sau auf Grund schlechter Mast- und Schlachtleistung (8\%), während bei den DE- und DL- Sauen das altersbedingte Ausscheiden (9 bzw. 10 \%) diesen Platz einnahm. Die Hauptselektionszeitpunkte für die unterschiedlichen Abgangsursachen waren bei der Zuchtleistung nach dem 4. Wurf, bei der Mast- und Schlachtleistung zwischen dem 2. und 5. Wurf, bei ausbleibender Trächtigkeit nach dem 1. und bei Krankheit bis zum 4. Wurf, während die Merzung wegen hohen Alters erst ab dem 5. bis 6. Wurf stattfand. Die Heritabilitätsschätzwerte der Abgangsursachen lagen für die Zuchtleistung zwischen $h^{2}=0,04$ und $h^{2}=0,08$, für die Mast- und Schlachtleistung zwischen $\mathrm{h}^{2}=0,01$ und $\mathrm{h}^{2}=0,09$, für Fruchtbarkeit bei $\mathrm{h}^{2}=0,02$, für Krankheit zwischen $\mathrm{h}^{2}=0,01$ und $\mathrm{h}^{2}=0,05$, für hohes Alter zwischen $\mathrm{h}^{2}=0,03$ und $\mathrm{h}^{2}=0,05$ und für sonstige Abgangsursachen zwischen $\mathrm{h}^{2}=0,05$ und $\mathrm{h}^{2}=0,16$.

Schlüsselwörter: Sau, Abgangsursache, Lebensleistung, Heritabilität

1.

\section{Einleitung}

Die Lebensleistung der Sau spiegelt sich in den Parametern Nutzungsdauer, Gesamtzahl im Leben einer Sau lebend geborener und aufgezogener Ferkel sowie der Ge- 
samtzahl Würfe wider. Mehrere Untersuchungen in den letzten Jahren haben gezeigt, dass Sauen in nahezu allen Zuchtstufen vor ihrem altersbedingten Leistungsmaximum abgehen. Die jährliche Abgangsrate liegt zwischen 40 und $50 \%$ (DAGORN und AUMAITRE, 1979; D’ALLAIRE et al., 1987; LUCIA et al, 1999; STEIN et al., 1990). Dadurch wird der Altersdurchschnitt der Sauen im Bestand zugunsten der Jungsauen verschoben, die weniger Würfe pro Jahr und weniger Ferkel pro Wurf erbringen, wodurch die Kosten pro aufgezogenem Ferkel stark ansteigen (DAGORN und AUMAITRE, 1979; DIJKHUIZEN et. al., 1986; KROES and VAN MALE, 1979; PATTISON et al., 1980). 15 - $46 \%$ des Sauenbestandes scheiden bereits nach dem ersten Wurf aus, während nur 29 - 57 \% maximal 3 Würfe erbringen. Die Hauptursachen für das frühzeitige Ausscheiden der Sauen aus den Betrieben sind zu 31-34 \% Fruchtbarkeitsprobleme der Sauen (DAGORN und AUMAITRE, 1979; D’ALLAIRE et al., 1987; DIJKHUIZEN et. al., 1986; EIKMEIER und MAYER, 1965; FINKE et al., 1984; LUCIA et al., 2000). Wegen geringer Zuchtleistung werden 6-24 \% und auf Grund von Beinschwächen 9 - 29 \% der Sauen gemerzt. Die Werte für den Anteil wegen hohen Alters abgegangener Sauen schwanken zwischen 8,7 - 27,2 \% (DAGORN und AUMAIRTE, 1979; D'ALLAIRE et al., 1987; DIJKHUIZEN et. al., 1986; LUCIA et al., 2000).

Ziel dieser Untersuchung ist es, die Abgangsursachen für Sauen der Rassen Deutsches Edelschwein, Deutsche Landrasse und Pietrain im Vergleich darzustellen und die Auswirkungen der verschiedenen Merzungsgründe auf die Lebensleistungsmerkmale Lebens- und Nutzungsdauer sowie Anzahl insgesamt lebend geborener bzw. aufgezogener Ferkel deutlich zu machen.

Des weiteren soll auf die Frage der Erblichkeit der Abgangsursachen eingegangen werden.

Material und Methoden

Das Landeskuratorium der Erzeugerringe für tierische Veredelung in Bayern e. V. (LKV) stellte den Datensatz für die nachfolgenden Auswertungen zur Verfügung. Das Datenmaterial erstreckte sich auf 46.932 zwischen 1989 bis 2002 in Bayern geborene Herdbuchsauen. Durch die im folgenden erläuterten Restriktionen wurden letztendlich 20.167 Sauen in der Analyse berücksichtigt. Das Datenmaterial wurde auf die Geburtsjahrgänge der Sauen von 1989 bis einschließlich 1994 beschränkt, damit alle Sauen die Chance hatten, eine vollständige Lebensleistung zu erbringen. Hierfür wurde die Lebens- und Nutzungsdauer mit dem zuletzt registrierten Datum der Sau berechnet. Die Nutzungsdauer in Tagen wird definiert als Zeitraum zwischen der ersten Abferkelung und dem Ausscheiden der Sau aus der Herde. Entsprechend errechnet sich die Lebensdauer in Tagen vom Geburtsdatum bis zum Abgangsdatum. Als insgesamt lebend geborene und aufgezogene Ferkel zählten die bis zum Ende des Beobachtungszeitraums erbrachten Ferkel. Die Anzahl insgesamt lebend geborener und aufgezogener Ferkel bezieht sich auf die gesamte Nutzungsdauer der Sau. Als lebend geboren gilt jedes lebende Ferkel zum Zeitpunkt der Geburt. Die Anzahl aufgezogener Ferkel bezieht sich auf die lebenden Tiere beim Absetzen von der Sau. Der Absetzzeitpunkt ist vom Management der Betriebe abhängig und daher variabel. Jedoch ist die Anzahl aufgezogener Ferkel nicht nur abhängig von der Anzahl lebend geborener Ferkel der Sau, sondern auch von der Anzahl zu- und umgesetzter Ferkel. Bei dieser Datenanalyse war es nicht möglich, die von der Sau aufgezogenen Ferkel des eigenen 
Wurfes von den zu- oder umgesetzten Tieren zu unterscheiden. TÖLLE et al. (1998) ermittelten bei einer Untersuchung von 44.643 Würfen der Rassen DL und DE einen maximalen Betriebsdurchschnitt der Anzahl umgesetzter Ferkel pro Wurf von 1,5 Ferkeln. Die Grundlage für die Auswertungen bildeten Herdbuchsauen der Rassen Deutsches Edelschwein (DE), Deutsche Landrasse (DL) und Pietrain (PI). Sauen anderer Rassen wurden auf Grund zu geringer Anzahlen nicht berücksichtigt. Das Erstferkelalter wurde auf ein Minimum von 305 Lebenstagen und ein Maximum von 500 Lebenstagen beschränkt, da darunter liegende und darüber liegende Werte nicht sinnvoll erschienen. Nur Sauen mit einer Mindestdauer der Zwischenwurfzeit von 136 Tagen wurden berücksichtigt. Die Mindestdauer der Zwischenwurfzeit von 136 Tagen ergab sich aus einer Tragedauer von $114 \pm 3$ Tagen, einer Säugezeit von 21 Tagen sowie der Zeitdauer von ca. 4 Tagen bis die Sau nach dem Absetzen der Ferkel wieder in Rausche kommt. Zwischenwurfzeiten über 420 Tagen wurden nicht mehr berücksichtigt, da man davon ausgehen kann, dass diese Sauen im Rohdatenmaterial falsch mitgeführt wurden. Sauen, für die bei der Anzahl lebend geborener Ferkel im ersten Wurf eine 0 eingetragen war und Tiere mit einem fiktiven Geburtsdatum wurden aus dem Datenmaterial entfernt. Unter den Punkt sonstige Restriktionen fielen die Sauen, bei denen die Angabe der Gesamtzahl lebend geborener Ferkel mit der aus den Wurfdaten errechneten Summe der lebend geborenen Ferkel nicht übereinstimmte oder eine nicht fortlaufende Wurfnummerierung eingetragen war (Tab. 1). 34 Sauen waren am Ende des Beobachtungszeitraum im Jahr 2002 noch nicht abgegangen und hatten daher auch keine Abgangsursache. Das in die Auswertung eingehende Datenmaterial enthielt somit 20.133 Sauen mit vollständigen Angaben. Die Gesamtzahl lässt sich wie folgt auf die drei Rassen aufteilen: 655 DE- Sauen, 13.005 DL-Sauen und 6.473 PI-Sauen (Tab. 2). Bei den Abgangsursachen der Sauen wurde zwischen geringer Zuchtleistung, geringer Mast- und Schlachtleistung, ausbleibender Trächtigkeit, Krankheit, Überzahl, zu hohes Alter und sonstige, nicht näher erläuterte Gründe unterschieden. Die Dokumentation der Abgangsgründe erfolgte nach den Angaben der Landwirte, so dürften teilweise unterschiedliche Definitionen dieser Parameter zu Grunde gelegen haben. Unter der Abgangsursache „Schlechte Zuchtleistung“ können sowohl die Wurfgröße, die Aufzuchtleistung, keine Rausche oder hohe Umrauscherquote sowie Verferkelung und ähnliches verstanden werden. Die geringe Mast- und Schlachtleistung bezieht sich auf die Ferkel der Sau und ihre Leistungen in der Mast, während unter dem Abgangsgrund „Ausbleibende Trächtigkeit“ sowohl Anöstrus als auch nicht beobachtete Rausche und vermehrtes Umrauschen der Sau verstanden werden kann. Unter die Abgangsursache „Krankheit“ fallen alle auftretenden Erkrankungen der Sau wie Fundamentprobleme, respiratorische, gastrointestinale oder urogenitale Infektionen genauso wie Mastitis, Metritis oder Uterusprolaps. Wegen der Abgangsursache „Überzählig“ gingen Sauen ab, die wegen zu großen Bestandsumfangs den Betrieb verlassen mussten, wobei bei der Merzungsentscheidung des Landwirts zumindest einer der anderen Abgangsgründe ebenfalls eine Rolle gespielt haben dürfte. Als „Zu hohes Alter“ kann sowohl das absolut zu hohe Alter der Sau für den Zuchteinsatz als auch das für den folgenden Wurfabschnitt zu weit fortgeschrittene Alter verstanden werden.

Um den Einfluss der unterschiedlichen Abgangsursachen auf die Lebensleistung der Sauen zu berechnen, wurde die varianzanalytische Auswertung der Merkmale Lebensalter, Nutzungsdauer, insgesamt im Leben einer Sau lebend geborene und insgesamt im Leben einer Sau aufgezogene Ferkel mit der Prozedur MIXED von SAS (Statistical 
Analysis Systems, Cary, NC, USA, 2003), Version 8.2, durchgeführt. Die im folgenden erläuterten Effekte wurden nach Rassen getrennt nach dem unten aufgeführten gemischten Modell geschätzt und auf Signifikanz geprüft. Bei der Analyse des Merkmals „Lebensalter“ wurde der Effekt „Erstferkelalter“ aus dem Modell entfernt.

\section{Tabelle 1}

Reduktion des Gesamtdatenbestandes durch eingeführte Restriktionen (Reduction of the whole data set by using restrictions)

\begin{tabular}{|c|c|}
\hline Restriktion & Sauenanzahl \\
\hline Rohdatenbestand & 46.932 \\
\hline Ausschluss aller Rassen außer DE, DL und PI & 601 \\
\hline fehlendes Geburtsdatum & 782 \\
\hline Anzahl lebend geborener Ferkel $=0$ & 149 \\
\hline $305>$ EFA $<500$ & 1.337 \\
\hline $136>$ ZWZ $<420$ & 57 \\
\hline Geburtsjahrgänge 1989 bis 1994 & - 21.959 \\
\hline Abgangsdatum $<$ maximales Wurfdatum & - $\quad 161$ \\
\hline ausgeschiedene Betriebe & 1.711 \\
\hline Sonstige Restriktionen & 8 \\
\hline Gesamtdatenbestand nach Reduktion & 20.167 \\
\hline
\end{tabular}

Modell 1 für die Analyse systematischer Effekte

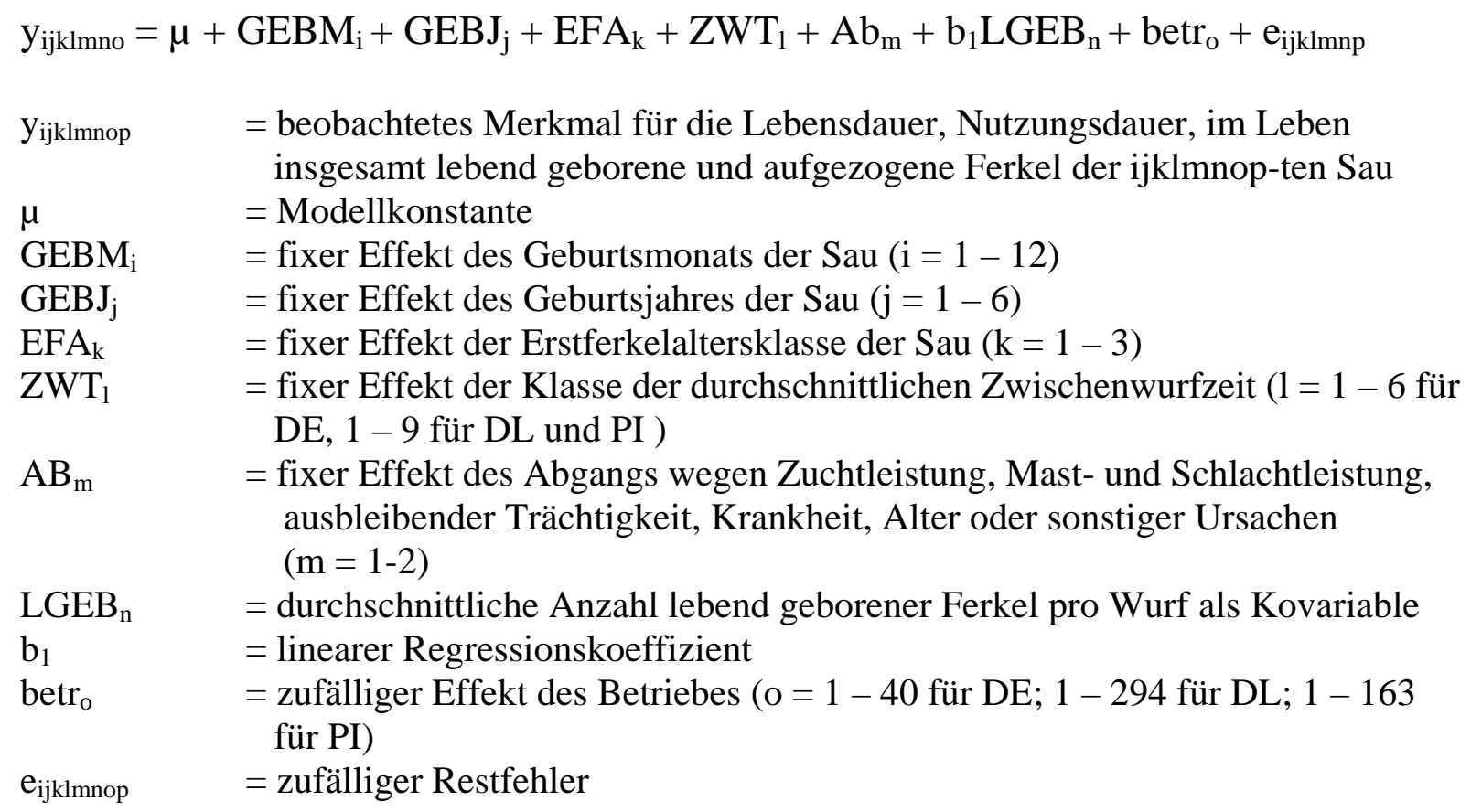

Das Erstferkelalter (EFA) der Sauen lag aufgrund der Restriktionen zwischen 305 und 500 Tagen und wurde in drei Klassen eingeteilt. Klasse 1 wurde mit Tieren besetzt, die vor dem 350. Lebenstag abferkelten. Klasse 2 beinhaltete Sauen, deren Erstferkelalter zwischen 350 und 380 Tagen lag, während sich in Klasse 3 nur Tiere befanden, die nach dem 380. Lebenstag den ersten Wurf erbrachten. Die Anzahl der Sauen innerhalb der Erstferkelaltersklassen betrug bei der Rasse DE zwischen 207 und 234, bei DL zwischen 3.173 und 5.391 und für die Rasse PI zwischen 1.362 und 2.673 Tieren.

Die durchschnittliche Zwischenwurfzeit (ZWT) ging ebenfalls als fixer Effekt in das Modell ein. Für die Sauen der Rassen DE und PI wurden 9 Klassen gebildet, während die DE-Sauen nur in 6 Klassen eingeteilt wurden, um eine zu geringe Klassenbeset- 
zung zu vermeiden. Klasse 1 enthielt die Tiere, die bereits nach dem ersten Wurf aus dem Betrieb ausschieden und für die somit keine Zwischenwurfzeit ermittelt werden konnte. Die Anzahl der Sauen in den Zwischenwurfzeitklassen schwankte bei DE zwischen 63 und 148, für die Rasse DL zwischen 685 und 2.690 und bei PI zwischen 166 und 1.505 Tieren. Die Effekte der unterschiedlichen Abgangsursachen wurden einzeln und nacheinander in das Modell eingebracht. Die durchschnittliche Anzahl lebend geborener Ferkel pro Wurf ergab sich aus der Gesamtzahl lebend geborener Ferkel und der Gesamtzahl Würfe, die die Sau in ihrem Leben erbracht hat.

Für die Abgangsursachen Zuchtleistung, Mast- und Schlachtleistung, ausbleibende Trächtigkeit, Krankheit, hohes Alter und sonstige Abgangsgründe als binäre Merkmale wurden die additiv-genetischen und residualen Varianzen mittels Residual Maximum Likelihood (REML) nach dem unten aufgeführten linearen Tiermodell geschätzt. Hierfür wurde das Programm VCE4, Version 4.2.5. (GROENEVELD, 1998) verwendet.

\section{Modell 2 für die Heritabilitätsschätzung}

$$
\begin{aligned}
& \mathrm{y}_{\mathrm{ijklmn}}=\mu+\mathrm{EFA}_{\mathrm{i}}+\mathrm{ZWT}_{\mathrm{j}}+\mathrm{b}_{1} \mathrm{LGEB}_{\mathrm{k}}+\mathrm{hjs}_{\mathrm{l}}+\mathrm{a}_{\mathrm{m}}+\mathrm{e}_{\mathrm{ijklmn}}
\end{aligned}
$$

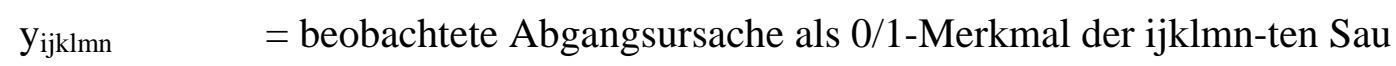

$$
\begin{aligned}
& \mu \quad=\text { Modellkonstante } \\
& \text { EFA } \left._{i} \quad \text { = fixer Effekt der Erstferkelaltersklasse der Sau ( } i=1-3\right) \\
& \mathrm{ZWT}_{\mathrm{j}} \quad=\text { fixer Effekt der Klasse der durchschnittlichen Zwischenwurfzeit }(\mathrm{j}=1-6 \text { für } \\
& \text { DE, } 1 \text { - } 9 \text { für DL und PI ) } \\
& \text { LGEB }_{\mathrm{k}} \quad=\text { durchschnittliche Anzahl lebend geborener Ferkel pro Wurf als Kovariable } \\
& \mathrm{b}_{1} \quad=\text { linearer Regressionskoeffizient } \\
& \text { hjs } 1 \text { = zufälliger Effekt der Herden-Jahr-Saison-Klasse }(\mathrm{l}=92 \text { für DE, } 732 \text { für DL, } \\
& 438 \text { für PI) } \\
& \mathrm{a}_{\mathrm{m}} \quad \text { = zufälliger additiv-genetischer Effekt des Tieres }(\mathrm{m}=1.026 \text { für } \mathrm{DE}, 21.271 \text { für } \\
& \text { DL, } 12.074 \text { für PI) }
\end{aligned}
$$

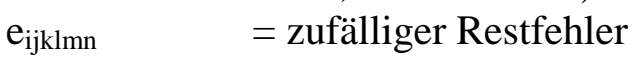

Das hier beschriebene Modell 2 unterschied sich von dem vorhergehenden durch die Einführung der Herden-Jahr-Saison-Klassen und des additiv-genetischen Effekts des Tieres. Der Effekt der Herden-Jahr-Saison-Klasse setzte sich aus dem Betrieb, dem Geburtsjahr und dem Geburtsmonat der Sau zusammen. Die Geburtsmonate wurden zu Quartalen zusammengefasst ( Januar bis März = Saison 1, April bis Juni = Saison 2; Juli bis September $=$ Saison 3, Oktober bis Dezember $=$ Saison 4).

Das für die 655 DE-Sauen erstellte Pedigree beinhaltete insgesamt 1.026 Tiere und ließ sich über 8 Generationen zurückverfolgen. Das Pedigree umfasste alle Mütter, war jedoch auf der väterlichen Seite nur zu 99 \% vollständig. Auf der Stufe der väterlichen Großeltern lag die Vollständigkeit bei nur $1 \%$, während sie auf der Stufe der mütterlichen Großeltern 95 \% erreichte. Die 655 in die Auswertung eingegangenen Sauen stammten von 106 Vätern und 227 Müttern ab. Auf der Basis von 13.008 DL-Sauen konnte ein Pedigree erstellt werden, das aus 21.271 Tieren bestand und 13 Generationen beinhaltete. Die Vollständigkeit des Pedigrees erreichte auf der Elternstufe 99 \%, auf der Stufe der väterlichen Großeltern 98 \% und der mütterlichen Großeltern 96 \%. Die DL-Sauen gingen auf 1.382 Väter und 5.829 Muttertiere zurück. Das Pedigree der PI-Sauen bestand aus 12.074 Tieren und konnte über 16 Generationen zurückverfolgt werden. Die Eltern waren zu 99 \% bekannt, während die väterlichen Großeltern eine 
Vollständigkeit von $98 \%$ und die mütterlichen Großeltern von 97\% erreichten. Die 6.481 PI-Sauen stammten von 1.087 Vätern und 3.029 Müttern ab.

\section{3. $\quad$ Ergebnisse}

\section{Analyse der Verteilung der Abgangsursachen}

Tabelle 2 stellt die Anzahl der abgegangenen Sauen und ihren prozentualen Anteil getrennt nach Abgangsursachen dar. Nach „Sonstigen Abgangsgründen“ schieden bei allen Rassen die meisten Tiere auf Grund ausbleibender Trächtigkeit aus (15\% bei DE- und PI-, 20 \% bei DL-Sauen), gefolgt von Krankheit mit 11 (DE) bis 13 \% (DL). Bei den DE- und DL-Tieren stand an dritter Stelle das Ausscheiden der Sau wegen hohen Alters mit 9 - $10 \%$, während sich hier bei den PI-Sauen mit $8 \%$ der Ausschluss wegen geringer Mast- und Schlachtleistung befand. Die geringste Anzahl DL-Sauen (2\%) schied auf Grund zu großen Umfangs des Gesamtbestandes aus, während die wenigsten DE-Tiere wegen geringer Mast- und Schlachtleistung gemerzt wurden. Bei den PI-Sauen hatte eine schlechte Zuchtleistung (4 \%) den geringsten Einfluss auf das Ausscheiden der Sau aus dem Betrieb.

Tabelle 2

Anzahl und Prozent (\%) der abgegangenen Sauen des gesamten Datenmaterials und nach Rassen getrennt pro Abgangsursache (Number and percentage (\%) of sows culled of the total data set and by breeds and culling reason)

\begin{tabular}{lcccccccc}
\hline Abgangsursache & \multicolumn{2}{c}{ Gesamt } & \multicolumn{2}{c}{ DE } & \multicolumn{2}{c}{ DL } & \multicolumn{2}{c}{ PI } \\
\hline geringe Zuchtleistung & 1.084 & $5 \%$ & 29 & $4 \%$ & 797 & $6 \%$ & 258 & $4 \%$ \\
geringe Mast-/Schlachtleist. & 914 & $5 \%$ & 10 & $2 \%$ & 397 & $3 \%$ & 507 & $8 \%$ \\
ausbleibende Trächtigkeit & 3.699 & $18 \%$ & 95 & $15 \%$ & 2.651 & $20 \%$ & 953 & $15 \%$ \\
Krankheit & 2.531 & $13 \%$ & 70 & $11 \%$ & 1.707 & $13 \%$ & 754 & $12 \%$ \\
überzählig & 685 & $3 \%$ & 15 & $2 \%$ & 247 & $2 \%$ & 423 & $7 \%$ \\
Alter & 1.574 & $8 \%$ & 66 & $10 \%$ & 1.148 & $9 \%$ & 360 & $6 \%$ \\
sonstige & 9.646 & $48 \%$ & 370 & $57 \%$ & 6.058 & $47 \%$ & 3.218 & $50 \%$ \\
\hline Gesamtanzahl & 20.133 & $100 \%$ & 655 & $100 \%$ & 13.005 & $100 \%$ & 6.473 & $100 \%$ \\
\hline
\end{tabular}

Tabelle 3

Frequenz (Anzahl und \%) der Sauen nach Anzahl erbrachter Würfe zum Zeitpunkt des Abgangs getrennt nach Rassen (Frequency (number and percentage) of sows by number of litters at the timepoint of culling by breeds)

\begin{tabular}{lccccccc}
\hline Rasse & \multicolumn{7}{c}{ Anzahl Würfe zum Zeitpunkt des Abgangs } \\
\hline \multirow{2}{*}{ DE } & 1 & 2 & 3 & 4 & $5-6$ & $7-8$ & $9+$ \\
\cline { 2 - 8 } & 129 & 92 & 84 & 73 & 25 & 79 & 73 \\
DL & $19,7 \%$ & $14,1 \%$ & $12,8 \%$ & $11,2 \%$ & $19,1 \%$ & $12,1 \%$ & $11,2 \%$ \\
& 2.690 & 1.862 & 1.592 & 1.434 & 2.482 & 1.649 & 1.296 \\
PI & $20,7 \%$ & $14,3 \%$ & $12,2 \%$ & $11,0 \%$ & $19,1 \%$ & $12,7 \%$ & $10,0 \%$ \\
& 1.503 & 1.258 & 919 & 761 & 1.035 & 628 & 369 \\
& $23,2 \%$ & $19,4 \%$ & $14,2 \%$ & $11,8 \%$ & $16,0 \%$ & $9,7 \%$ & $5,7 \%$ \\
\hline
\end{tabular}

$\mathrm{DE}=$ Deutsches Edelschwein, $\mathrm{DL}=$ Deutsche Landrasse, $\mathrm{PI}=$ Pietrain

Die Abgangsrate nach dem ersten Wurf war für alle drei Rassen am höchsten. Bei den DE- und DL-Sauen gingen ca. 20 \% zu diesem Zeitpunkt ab, während 23 \% der PISauen nach dem ersten Abferkeln den Betrieb verließen (Tab. 3). Die zweithöchste Abgangsrate fand sich mit $19 \%$ bei den Rassen DE und DL nach dem 5. bis 6. Wurf. Die PI-Sauen hingegen gingen mit $19 \%$ am zweithäufigsten nach dem 2. Wurf ab, während der geringste Prozentsatz an Sauen der drei Rassen nach dem 9. Wurf ausschied. Nur 42 \% der DE- und DL-Tiere und nur $32 \%$ der PI-Sauen erbrachten mehr als 4 Würfe. 
Bei allen drei Rassen lag die durchschnittliche Lebensleistung für die Tiere, die wegen hohen Alters ausschieden, am höchsten, während sie die geringste Leistung mit der Abgangsursache Mast- und Schlachtleistung und Ausscheidens wegen Erkrankung erbrachten (Tab. 4). Die höchsten Mittelwerte der Lebens- und Nutzungsdauer mit 1.024 und 652 Tagen sowie der Anzahl insgesamt lebend geborener bzw. aufgezogener Ferkel mit 43,0 bzw. 39,4 wurde für die Abgangsursache „Zuchtleistung“ bei den DE-Sauen erreicht. Für alle weiteren Abgangsursachen, außer dem altersbedingten Abgang und sonstiger Abgangsgründe, waren die Mittelwerte für die DE-Sauen ebenfalls am höchsten. DE-Sauen mit dem Abgangsgrund „Mast- und Schlachtleistung“ lebten 872 Tage, erreichten eine Nutzungsdauer von 496 Tagen, gebaren insgesamt 34,5 lebende Ferkel und zogen davon 33,3 Ferkel auf. Bei ausbleibender Trächtigkeit erreichten die Sauen eine Lebens- und Nutzungsdauer von 954 und 590 Tagen und eine Anzahl insgesamt lebend geborener bzw. aufgezogener Ferkel von 39,6 bzw. 37,1. Für DE-Sauen mit krankheitsbedingtem Abgang wurden Werte für die Lebensund Nutzungsdauer von 871 und 502 Tagen und für die Anzahl insgesamt lebend geborener bzw. aufgezogener Ferkel von 34,8 bzw. 32,2 berechnet. Überzählige DESauen erreichten eine Lebens- und Nutzungsdauer von 718 und 356 Tagen, während die Anzahl insgesamt lebend geborener bzw. aufgezogener Ferkel 26,0 bzw. 24,3 betrug. Für den Abgangsgrund „Zu hohes Alter“ wurden die höchsten Mittelwerte mit 1.732 und 1.360 Tagen für die Lebens- und Nutzungsdauer und mit 82,3 bzw. 76,4 Ferkeln für die Anzahl insgesamt lebend geborener bzw. aufgezogener Ferkel bei den

Tabelle 4

Mittelwerte und ihre Standardfehler der Merkmale Lebensdauer, Nutzungsdauer (in Tagen), insgesamt lebend geborene und aufgezogene Ferkel für die verschiedenen Abgangsursachen getrennt nach Rassen (Means and standard deviations of longevity, length of productive life (in days), number of piglets born alive and number of weaned piglets in total lifetime by culling reasons and breeds)

\begin{tabular}{lccccc}
\hline Abgangsursache / Rasse & $\mathrm{n}$ & $\begin{array}{c}\text { Lebensdauer } \\
\text { (Tage) }\end{array}$ & $\begin{array}{c}\text { Nutzungsdauer } \\
\text { (Tage) }\end{array}$ & $\begin{array}{c}\text { insgesamt leb. } \\
\text { geb. Ferkel }\end{array}$ & $\begin{array}{c}\text { insgesamt } \\
\text { aufgez. Ferkel }\end{array}$ \\
\hline $\begin{array}{l}\text { Deutsches Edelschwein } \\
\text { Zuchtleistung }\end{array}$ & 29 & $1.024 \pm 539$ & $652 \pm 543$ & $43,0 \pm 36,7$ & $39,4 \pm 33,7$ \\
Mast-/Schlachtleistung & 10 & $872 \pm 254$ & $496 \pm 258$ & $34,5 \pm 15,0$ & $33,3 \pm 14,6$ \\
ausbleib. Trächtigkeit & 95 & $954 \pm 386$ & $590 \pm 393$ & $39,6 \pm 26,4$ & $37,1 \pm 25,0$ \\
Krankheit & 70 & $871 \pm 377$ & $502 \pm 373$ & $34,8 \pm 23,2$ & $32,2 \pm 21,3$ \\
überzählige & 15 & $718 \pm 338$ & $356 \pm 342$ & $26,0 \pm 21,7$ & $24,3 \pm 20,8$ \\
Alter & 66 & $1.679 \pm 426$ & $1.321 \pm 421$ & $94,5 \pm 30,3$ & $85,7 \pm 26,5$ \\
sonstige & 370 & $1.065 \pm 508$ & $689 \pm 507$ & $43,5 \pm 32,5$ & $40,1 \pm 29,4$ \\
\hline Deutsche Landrasse & & & & & \\
Zuchtleistung & 797 & $895 \pm 355$ & $533 \pm 355$ & $32,8 \pm 21,9$ & $30,1 \pm 20,4$ \\
Mast-/Schlachtleistung & 397 & $854 \pm 294$ & $496 \pm 292$ & $33,7 \pm 19,2$ & $31,6 \pm 18,0$ \\
ausbleib. Trächtigkeit & 2651 & $925 \pm 412$ & $563 \pm 412$ & $34,6 \pm 26,0$ & $32,4 \pm 24,3$ \\
Krankheit & 1707 & $865 \pm 379$ & $505 \pm 379$ & $34,4 \pm 24,3$ & $32,1 \pm 22,6$ \\
überzählige & 247 & $1.030 \pm 448$ & $671 \pm 444$ & $43,5 \pm 26,2$ & $40,8 \pm 24,5$ \\
Alter & 1148 & $1.731 \pm 369$ & $1.372 \pm 369$ & $91,3 \pm 24,4$ & $85,2 \pm 22,8$ \\
sonstige & 6058 & $1.072 \pm 497$ & $710 \pm 496$ & $43,7 \pm 29,8$ & $41,0 \pm 28,0$ \\
\hline Pietrain & & & & & \\
Zuchtleistung & 258 & $897 \pm 414$ & $523 \pm 410$ & $30,2 \pm 20,6$ & $27,6 \pm 19,5$ \\
Mast-/Schlachtleistung & 507 & $848 \pm 305$ & $471 \pm 306$ & $29,6 \pm 16,6$ & $27,7 \pm 15,6$ \\
ausbleib.Trächtigkeit & 953 & $887 \pm 376$ & $513 \pm 372$ & $27,8 \pm 21,0$ & $25,8 \pm 19,6$ \\
Krankheit & 754 & $794 \pm 350$ & $422 \pm 349$ & $26,4 \pm 19,2$ & $24,5 \pm 17,8$ \\
überzählige & 423 & $1.008 \pm 402$ & $628 \pm 401$ & $38,8 \pm 23,0$ & $36,5 \pm 21,7$ \\
Alter & 360 & $1.732 \pm 404$ & $1.360 \pm 404$ & $82,3 \pm 23,8$ & $76,4 \pm 22,8$ \\
sonstige & 3218 & $1.032 \pm 503$ & $655 \pm 500$ & $36,1 \pm 25,6$ & $33,6 \pm 23,8$ \\
\hline
\end{tabular}


PI-Sauen ermittelt. Die höchsten Mittelwerte für die sonstigen Abgangsgründe wurden bei der Rasse DL errechnet. Diese DL-Sauen lebten 1.072 Tage, erreichten eine Nutzungsdauer von 710 Tagen, gebaren insgesamt 47,7 lebende Ferkel und zogen davon 41,0 Ferkel auf.

Tabelle 5

Frequenz (\%) der Abgangsursachen nach Anzahl erbrachter Würfe zum Zeitpunkt des Abgangs (Frequency (\%) of culling reasons by number of litters at the timepoint of culling)

\begin{tabular}{|c|c|c|c|c|c|c|c|}
\hline \multirow[t]{2}{*}{ Abgangsursache/ Rasse } & \multicolumn{7}{|c|}{ Anzahl Würfe zum Zeitpunkt des Abgangs } \\
\hline & 1 & 2 & 3 & 4 & $5-6$ & $7-8$ & $9+$ \\
\hline \multicolumn{8}{|l|}{ Deutsches Edelschwein } \\
\hline Zuchtleistung & 4,7 & 5,4 & 2,4 & 6,9 & 4,0 & 2,5 & 5,5 \\
\hline Mast-Schlachtleist. & 0,0 & 4,4 & 1,2 & 5,5 & 0,8 & 0,0 & 0,0 \\
\hline ausbl. Trächtigkeit & 17,8 & 12,0 & 22,6 & 12,3 & 13,6 & 15,2 & 5,5 \\
\hline Krankheit & 12,4 & 19,4 & 10,7 & 9,6 & 12,0 & 6,3 & 2,7 \\
\hline überzählig & 4,6 & 4,4 & 1,2 & 2,7 & 0,8 & 1,3 & 0,0 \\
\hline Alter & 0,0 & 0,0 & 0,0 & 2,7 & 11,2 & 21,5 & 45,2 \\
\hline sonstige & 60,5 & 56,5 & 61,9 & 60,3 & 57,6 & 53,2 & 41,1 \\
\hline \multicolumn{8}{|l|}{ Deutsche Landrasse } \\
\hline Zuchtleistung & 5,2 & 9,0 & 7,4 & 7,5 & 7,1 & 4,2 & 1,4 \\
\hline Mast-Schlachtleist. & 1,9 & 5,3 & 6,2 & 4,1 & 3,0 & 0,7 & 0,2 \\
\hline ausbl. Trächtigkeit & 29,7 & 23,3 & 21,0 & 21,1 & 18,9 & 13,5 & 7,0 \\
\hline Krankheit & 16,7 & 16,3 & 16,4 & 14,2 & 12,2 & 9,0 & 3,0 \\
\hline überzählig & 1,6 & 1,6 & 2,2 & 2,0 & 2,9 & 1,3 & 1,2 \\
\hline Alter & 0,2 & 0,1 & 0,1 & 1,1 & 5,0 & 23,7 & 46,9 \\
\hline sonstige & 44,7 & 44,4 & 46,8 & 50,0 & 50,9 & 47,6 & 40,2 \\
\hline \multicolumn{8}{|l|}{ Pietrain } \\
\hline Zuchtleistung & 4,0 & 4,0 & 4,9 & 5,8 & 4,0 & 1,4 & 2,4 \\
\hline Mast-Schlachtleist. & 2,8 & 15,4 & 13,0 & 10,8 & 4,4 & 3,2 & 1,1 \\
\hline ausbl. Trächtigkeit & 21,4 & 15,6 & 15,6 & 13,4 & 12,2 & 8,1 & 3,5 \\
\hline Krankheit & 17,0 & 14,8 & 10,1 & 11,4 & 8,7 & 5,4 & 2,4 \\
\hline überzählig & 4,5 & 5,5 & 6,4 & 7,9 & 9,2 & 9,1 & 4,1 \\
\hline Alter & 0,1 & 0,2 & 0,7 & 1,5 & 4,4 & 21,3 & 43,4 \\
\hline sonstige & 50,2 & 44,6 & 49,4 & 49,3 & 57,1 & 51,4 & 43,1 \\
\hline
\end{tabular}

Tabelle 5 stellt die unterschiedliche Verteilung (in \%) der einzelnen Abgangsgründe auf die verschiedenen Abgangszeiten der Sauen dar. Für die Würfe 1 bis 4 wurden die Abgangsursachen getrennt dargestellt, während die Würfe 5-6, 7-8 und mehr als 9 Würfe in Klassen zusammengefasst wurden, da keine nennenswerten Veränderungen innerhalb dieser Klassen stattfand. Bei allen drei Rassen war der Hauptabgangsgrund vom ersten bis zum sechsten Wurf ausbleibende Trächtigkeit, ab dem siebten Wurf schieden die meisten Sauen wegen hohen Alters aus. Die im folgenden erwähnten Prozentangaben beziehen sich auf die Gesamtzahl Sauen, die in den beschriebenen Wurfgruppen abgingen. Bei den DE-Sauen hatte die Zuchtleistung nach dem 4. Wurf den größten Einfluss auf die Merzung der Sau (6,9 \%), während sie nach dem 3. und 7.-8. Wurf mit 2,4 und 2,5 \% die geringste Bedeutung erlangte. Die Mast- und Schlachtleistung erreichte mit 5,5\% ihr Maximum als Abgangsgrund nach dem 4. Wurf und ihren geringsten prozentualen Anteil nach dem 1. und nach dem 7. Wurf (0\%). Die Abgangsursache „Krankheit“ spielte während der ersten 6 Würfe eine große Rolle bei der Merzung der Sauen (9,6 bis 19,4 \%), verlor jedoch später an Bedeutung. Wegen zu großer Bestandsgröße gingen die meisten Sauen nach den ersten beiden Würfen ab (4,4 bis $4,6 \%)$.

Die Zuchtleistung hatte bei der Rasse DL ihren größten Einfluss auf den Abgang der Sauen vom 2. bis zum 6. Wurf (7,1 bis 9,0\%) und war für das Ausscheiden nach über 
8 Würfen mit 1,4 \% nahezu bedeutungslos. Der Abgangsgrund „Geringe Mast- und Schlachtleistung“ erreichte seinen höchsten prozentualen Anteil nach dem 3. Wurf (6,2 \%) und fiel in den folgenden Würfen bis auf 0,2 \% ab. „Krankheit“ hatte während der ersten 6 Würfe einen relativ gleichbleibend hohen Einfluss auf die Merzung der Sauen (12,2 bis 16,7 \%), verlor danach jedoch an Bedeutung. Wegen zu großer Bestandsgröße schieden nach dem 5. bis 6. Wurf die meisten Sauen aus (2,9\%), während dieser Abgangsgrund die geringste Bedeutung beim Ausscheiden der Sauen über dem 9. Wurf hatte.

Die Zuchtleistung spielte bei der Merzung der PI-Sauen nach dem 4. Wurf die größte Rolle (5,8 \%), war jedoch nach dem 7. bis 8. Wurf mit 1,4\% nahezu bedeutungslos. Die Mast- und Schlachtleistung beeinflusste den Abgang der Sauen vom 2. bis zum 4. Wurf mit 10,8 bis 15,4 \% am stärksten, während dieser Abgangsgrund nach dem 9. Wurf nur noch 1,1\% erreichte. Wie bei den DL-Sauen, hatte „Krankheit“ als Ursache für den Abgang der PI-Sauen während der ersten 6 Würfe mit 10,1 bis 17,0 \% einen relativ gleichbleibend hohen Einfluss, der jedoch im folgenden bis auf 2,4 \% stark abfiel. Wegen zu großer Bestandsgröße schieden mit etwa 9 \% die meisten PI-Sauen nach dem 5. bis 8. Wurf aus.

Tabelle 6

Signifikante Einflüsse (Irrtumswahrscheinlichkeiten) der verschiedenen Abgangsursachen auf die Merkmale Lebens- und Nutzungsdauer sowie der Anzahl insgesamt lebend geborener und aufgezogener Ferkel (Significant influences (error probabilities) of the different culling reasons on longevity, length of productive life, number of piglets born alive and number of weaned piglets in total lifetime)

\begin{tabular}{|c|c|c|c|c|}
\hline Rasse / Effekt & Lebensdauer & Nutzungsdauer & $\begin{array}{l}\text { insgesamt lebend } \\
\text { geborene Ferkel }\end{array}$ & $\begin{array}{c}\text { insgesamt } \\
\text { aufgezogene } \\
\text { Ferkel }\end{array}$ \\
\hline \multicolumn{5}{|l|}{ Deutsches Edelschwein } \\
\hline $\begin{array}{l}\text { Zuchtleistung } \\
\text { Mast-/Schlachtleistung } \\
\text { ausbl. Trächtigkeit } \\
\text { Krankheit } \\
\text { hohes Alter } \\
\text { sonstige }\end{array}$ & $\begin{array}{c}0,895 \\
0,010 \\
0,263 \\
<0,001 \\
<0,001 \\
0,034\end{array}$ & $\begin{array}{c}0,863 \\
0,011 \\
0,256 \\
<0,001 \\
<0,001 \\
0,033\end{array}$ & $\begin{array}{c}0,905 \\
0,032 \\
0,063 \\
0,002 \\
<0,001 \\
0,004\end{array}$ & $\begin{array}{c}0,947 \\
0,049 \\
0,069 \\
0,002 \\
<0,001 \\
0,004\end{array}$ \\
\hline \multicolumn{5}{|l|}{ Deutsche Landrasse } \\
\hline $\begin{array}{l}\text { Zuchtleistung } \\
\text { Mast-/Schlachtleistung } \\
\text { ausbl. Trächtigkeit } \\
\text { Krankheit } \\
\text { hohes Alter } \\
\text { Sonstige }\end{array}$ & $\begin{array}{c}<0,001 \\
<0,001 \\
<0,001 \\
<0,001 \\
<0,001 \\
0,650\end{array}$ & $\begin{array}{c}<0,001 \\
<0,001 \\
<0,001 \\
<0,001 \\
<0,001 \\
0,620\end{array}$ & $\begin{array}{c}<0,001 \\
<0,001 \\
<0,001 \\
<0,001 \\
<0,001 \\
0,003\end{array}$ & $\begin{array}{c}<0,001 \\
<0,001 \\
<0,001 \\
<0,001 \\
<0,001 \\
0,005\end{array}$ \\
\hline \multicolumn{5}{|l|}{ Pietrain } \\
\hline $\begin{array}{l}\text { Zuchtleistung } \\
\text { Mast-/Schlachtleistung } \\
\text { ausbl. Trächtigkeit } \\
\text { Krankheit } \\
\text { hohes Alter } \\
\text { sonstige }\end{array}$ & $\begin{aligned} & 0,012 \\
< & 0,001 \\
< & 0,001 \\
< & 0,001 \\
< & 0,001 \\
< & 0,001\end{aligned}$ & $\begin{aligned} & 0,001 \\
< & 0,001 \\
< & 0,001 \\
< & 0,001 \\
< & 0,001 \\
< & 0,001\end{aligned}$ & $\begin{array}{c}0,066 \\
<0,001 \\
<0,001 \\
<0,001 \\
<0,001 \\
0,500\end{array}$ & $\begin{array}{c}0,028 \\
<0,001 \\
<0,001 \\
<0,001 \\
<0,001 \\
0,472\end{array}$ \\
\hline
\end{tabular}

Effekt der Abgangsursachen auf die Lebensleistung

In Tabelle 6 sind die Ergebnisse der Varianzanalyse mit ihren Irrtumswahrscheinlichkeiten für die Signifikanz der Effekte der unterschiedlichen Abgangsursachen auf die Merkmale „Lebens- und Nutzungsdauer“ sowie „Insgesamt lebend geborene und aufgezogene Ferkel“ getrennt nach Rassen aufgeführt. Bei den DE-Sauen hatten die Abgangsursachen „Zuchtleistung“ und „Ausbleibende Trächtigkeit“ keinen 
signifikanten Einfluss auf die analysierten Merkmale. Dagegen beeinflussten die Abgangsgründe „Mast- und Schlachtleistung“, „Krankheit“, „Hohes Alter“ und „Sonstige Ursachen“ alle Merkmale signifikant. Bei den DL-Sauen hatten alle Abgangsursachen auf die vier Merkmale, mit Ausnahme der "Sonstige Abgangsgründe“ bei den Merkmalen „Lebens- und Nutzungsdauer“, einen signifikanten Einfluss. Bei der Rasse PI beeinflusste die Zuchtleistung alle Merkmale signifikant, außer der Anzahl insgesamt lebend geborener Ferkel, bei dem der Wert knapp über der Signifikanzgrenze lag. Die Anzahl insgesamt im Leben der Sau lebend geborener und aufgezogener Ferkel wurde durch die sonstigen Abgangsgründe nicht signifikant beeinflusst, während im übrigen alle weiteren Abgangsursachen auf alle analysierten Merkmale signifikanten Einfluss hatten.

Tabelle 7

LS-Mittelwerte und deren Standardfehler für die Lebensdauer, Nutzungsdauer (in Tagen), insgesamt lebend geborene und aufgezogene Ferkel nach Abgangsursachen (LS-means and their standard errors for longevity, length of productive life (in days), number of piglets born alive and number of weaned piglets in total lifetime by culling reasons)

\begin{tabular}{|c|c|c|c|c|}
\hline $\begin{array}{l}\text { Geburtsjahr } \\
\text { der Sau }\end{array}$ & $\begin{array}{c}\text { Lebensdauer } \\
\text { (Tage) }\end{array}$ & $\begin{array}{l}\text { Nutzungsdauer } \\
\text { (Tage) }\end{array}$ & $\begin{array}{c}\text { insgesamt } \\
\text { lebend geborene } \\
\text { Ferkel }\end{array}$ & $\begin{array}{l}\text { insgesamt } \\
\text { aufgezogene } \\
\text { Ferkel }\end{array}$ \\
\hline \multicolumn{5}{|l|}{ Deutsches Edelschwein } \\
\hline Zuchtleistung & $1079 \pm 85$ & $704 \pm 86,1$ & $45,4 \pm 5,5$ & $42,4 \pm 5,0$ \\
\hline Mast-/Schlachtleistung & $759 \pm 133$ & $391 \pm 134,2$ & $28,2 \pm 8,7$ & $27,8 \pm 7,9$ \\
\hline ausbl. Trächtigkeit & $1043 \pm 56$ & $673 \pm 55,9$ & $41,1 \pm 3,6$ & $38,3 \pm 3,3$ \\
\hline Krankheit & $916 \pm 60$ & $549 \pm 60,1$ & $36,5 \pm 3,8$ & $34,2 \pm 3,5$ \\
\hline hohes Alter & $1597 \pm 59$ & $1233 \pm 59$ & $83,2 \pm 3,7$ & $75,6 \pm 3,4$ \\
\hline sonstige & $1053 \pm 43$ & $684 \pm 43$ & $42,8 \pm 2,7$ & $39,8 \pm 2,5$ \\
\hline \multicolumn{5}{|l|}{ Deutsches Landrasse } \\
\hline Zuchtleistung & $1005 \pm 18$ & $673 \pm 18,0$ & $39,6 \pm 1,0$ & $36,6 \pm 1,0$ \\
\hline Mast-/Schlachtleistung & $881 \pm 23$ & $515 \pm 22,6$ & $32,2 \pm 1,3$ & $30,4 \pm 1,2$ \\
\hline ausbl. Trächtigkeit & $1045 \pm 13$ & $680 \pm 13,4$ & $39,3 \pm 0,7$ & $36,9 \pm 0,7$ \\
\hline Krankheit & $953 \pm 15$ & $588 \pm 14,7$ & $37,4 \pm 0,8$ & $35,1 \pm 0,8$ \\
\hline hohes Alter & $1670 \pm 15$ & $1306 \pm 15$ & $81,5 \pm 0,8$ & $76,2 \pm 0,8$ \\
\hline sonstige & $1112 \pm 12$ & $746 \pm 12$ & $44,5 \pm 0,6$ & $41,7 \pm 0,6$ \\
\hline \multicolumn{5}{|l|}{ Pietrain } \\
\hline Zuchtleistung & $975 \pm 28$ & $603 \pm 28,1$ & $35,0 \pm 1,4$ & $32,1 \pm 1,3$ \\
\hline Mast-/Schlachtleistung & $829 \pm 22$ & $456 \pm 22,4$ & $27,1 \pm 1,1$ & $25,3 \pm 1,0$ \\
\hline ausbl. Trächtigkeit & $989 \pm 19$ & $614 \pm 19,3$ & $33,3 \pm 0,9$ & $31,0 \pm 0,9$ \\
\hline Krankheit & $897 \pm 21$ & $523 \pm 20,6$ & $31,5 \pm 1,0$ & $29,4 \pm 0,9$ \\
\hline hohes Alter & $1633 \pm 24$ & $1260 \pm 24$ & $74,0 \pm 1,1$ & $68,6 \pm 1,1$ \\
\hline sonstige & $1058 \pm 16$ & $684 \pm 16$ & $37,5 \pm 0,7$ & $34,8 \pm 0,7$ \\
\hline
\end{tabular}

Tabelle 7 gibt die LS-Mittelwerte der Merkmale für die verschiedenen Abgangsursachen nach Rassen getrennt an. Die Abgangsursache „Hohes Alter“ erreichte für die drei Rassen die höchsten Werte für alle Merkmale, gefolgt von Fruchtbarkeitsproblemen bei den DL- und PI-Sauen, während bei den DE-Sauen die Zuchtleistung an zweiter Stelle stand. Sauen, die wegen schlechter Mast- und Schlachtleistung abgingen, hatten bei allen Rassen die geringsten LS-Mittelwerte für die Merkmale der Lebensleistung. Die Abgangsursache „Zuchtleistung“ erbrachte bei den DE-Sauen die höchsten LS-Mittelwerte für die Lebensleistung mit 1.079 und 704 Tagen für die Lebens- und Nutzungsdauer sowie mit 45,4 bzw. 42,4 insgesamt lebend geborenen bzw. aufgezogenen Ferkeln. Für alle anderen Abgangsursachen waren die LS-Mittelwerte bei den DL-Sauen am höchsten. Sauen, die wegen schlechter Mast- und Schlachtleis- 
tung abgingen, erreichten die höchsten Werte für die Lebens- und Nutzungsdauer von 881 und 515 Tagen, während die Anzahl insgesamt lebend geborener bzw. aufgezogener Ferkel 32,2 bzw. 30,4 betrug. Die LS-Mittelwerte für die Abgangsursache „Ausbleibende Trächtigkeit“ waren mit 1.045 und 680 Tagen am höchsten, während die Anzahl insgesamt lebend geborener bzw. aufgezogener Ferkel einen Wert von 39,2 bzw. 36,9 erreichte. DL-Sauen mit der Abgangsursache „Zu hohes Alter“ erbrachten eine Lebens- und Nutzungsdauer von 1.670 und 1.306 Tagen, gebaren insgesamt 81,5 lebende Ferkel und zogen davon 76,2 Ferkel auf. Die LS-Mittelwerte für die sonstigen Abgangsursachen waren mit 1.112 und 746 Tagen für die Lebens- und Nutzungsdauer und mit 44,5 und 41,7 Ferkeln für die Anzahl insgesamt lebend geborener und aufgezogener Ferkel am höchsten. Bei den PI-Sauen waren die errechneten Werte für alle Abgangsursachen am geringsten.

Tabelle 8

Mittels linearer Tiermodelle univariat geschätzte Heritabilitäten $\left(\mathrm{h}^{2}\right)$ und deren Standardfehler (SE) für die unterschiedlichen Abgangsursachen für die Rassen DE, DL und PI (Heritability estimates $\left(\mathrm{h}^{2}\right)$ and their standard errors (SE) of the different culling reasons using an univariate linear animal model for the breeds DE, DL and PI)

\begin{tabular}{lccc}
\hline Abgangsursache & \multicolumn{3}{c}{ Rasse } \\
\cline { 2 - 4 } & $\mathrm{DE}$ & $\mathrm{DL}$ & $\mathrm{PI}$ \\
& $\mathrm{h}^{2} \pm \mathrm{SE}$ & $\mathrm{h}^{2} \pm \mathrm{SE}$ & $\mathrm{h}^{2} \pm \mathrm{SE}$ \\
\hline Zuchtleistung & $0,078 \pm 0,043$ & $0,044 \pm 0,008$ & $0,062 \pm 0,014$ \\
Mast-/Schlachtleistung & $0,013 \pm 0,024$ & $0,056 \pm 0,010$ & $0,085 \pm 0,015$ \\
ausbleibende Trächtigkeit & nicht schätzbar & $0,019 \pm 0,007$ & $0,015 \pm 0,009$ \\
Krankheit & $0,054 \pm 0,042$ & $0,044 \pm 0,009$ & $0,013 \pm 0,009$ \\
Alter & $0,036 \pm 0,031$ & $0,029 \pm 0,007$ & $0,054 \pm 0,010$ \\
sonstige & $0,159 \pm 0,059$ & $0,047 \pm 0,009$ & $0,051 \pm 0,012$ \\
\hline
\end{tabular}

Heritabilitätsschätzung

Aus Tabelle 8 ist zu entnehmen, dass die Heritabilitätsschätzwerte für die Abgänge wegen ungenügender Zuchtleistung zwischen $h^{2}=0,04$ (DL) und $h^{2}=0,08$ (DE) lagen. Die Merzung wegen ungenügender Mast- und Schlachtleistung erreichte Werte von $h^{2}=0,01$ (DE) bis $h^{2}=0,09(\mathrm{PI})$. Während die Schätzwerte für die Abgangsursache „Ausbleibende Trächtigkeit“ bei den DL- und PI-Sauen $\mathrm{h}^{2}=0,02$ betrugen, konnte für die Rasse DE keine Heritabilität geschätzt werden. Für die Abgangsursache „Krankheit“ konnten Werte von $\mathrm{h}^{2}=0,01$ (für PI) bis $\mathrm{h}^{2}=0,05$ (für DE) ermittelt werden. Die Heritabilitätsschätzwerte für das altersbedingte Ausscheiden der Sau betrugen zwischen $\mathrm{h}^{2}=0,03$ (DL) und $\mathrm{h}^{2}=0,05$ (PI), während die sonstigen Abgangsgründe Werte von 0,05 bis 0,16 erreichten. Bei den Sauen der Rasse DE wurden die höchsten Schätzwerte für die Abgangsursachen „Sonstige Gründe“ und „Ungenügende Zuchtleistung“ gefunden. Die geringsten Werte wurden für die Abgänge wegen geringer Mast- und Schlachtleistung ermittelt, während hierfür bei den PI- und DL-Sauen die Heritabilitätswerte am höchsten lagen. Die geringsten Heritabilitäten wurden bei den DL-Sauen für Merzungen wegen ausbleibender Trächtigkeit und bei den PI-Sauen für Abgänge wegen Krankheit geschätzt.

4. Diskussion

Abgangsursachen allgemein

Die hier gefundene Gewichtung der Abgangsursachen mit Fruchtbarkeitsproblemen an erster und Krankheit an zweiter Stelle betraf die drei untersuchten Rassen gleicherma- 
ßen. Während jedoch bei den DE- und DL-Sauen an dritter Stelle Merzung wegen hohen Alters stand, wurden die PI-Sauen am dritthäufigsten wegen schlechter Mast- und Schlachtleistung aus der Zucht ausgeschlossen, da dieser Aspekt bei der Vaterrasse einen deutlich höheren Stellenwert hat als bei den Mutterlinien. Bei allen übrigen Abgangsursachen ergaben sich keine nennenswerten Unterschiede zwischen den Rassen. EIKMEIER und MAYER (1965) beobachteten 171 Sauen aus 67 hessischen Herdbuchbetrieben. Sie ermittelten Sterilität mit 34\% als häufigste Abgangsursache gefolgt von unbefriedigender Zuchtleistung (24\%), was von den hier errechneten Werten von 18 bzw. 5 \% deutlich nach oben abweicht. Dies mag sowohl mit der unterschiedlichen Anzahl untersuchter Sauen und der unterschiedlichen Definition der Zuchtleistung als auch mit dem zeitlichen Abstand der beiden Untersuchungen zusammenhängen. Bei einer Untersuchung von DAGORN und AUMAITRE (1979) an französischen Sauen aus kommerziellen Herden schieden bereits 21,2 \% der Sauen nach dem ersten Wurf aus, was mit den hier ermittelten Werten von 19,7 -23,2 \% übereinstimmt. Die Reihenfolge der Hauptabgangsgründe weicht jedoch von der hier gefundenen ab: Hauptgründe der Merzungen waren bei DAGORN und AUMAITRE (1979) ausbleibende Trächtigkeit mit 31\%, altersbedingter Rückgang der Produktivität mit 27\% und Beinschwächen mit 8,8\%, gefolgt von geringer Wurf- und Aufzuchtleistung mit 8,4\% bei 105.454 Sauen der Rassen Large White, französische Landrasse und Kreuzungen aus diesen beiden. FINKE et al. (1984) untersuchten 78 Reinzuchtsauen der Rassen DL und der Deutschen Landrasse B sowie 41 Kreuzungssauen $(\mathrm{DE} \times \mathrm{DL})$ auf Zuchtleistungsmerkmale, von denen $43 \%$ vor dem 4 . Wurf ausschieden, was nur geringgradig unter den hier gefundenen Werten für DE- und DL-Sauen von 46,6 - 47,2 \% lag. Jedoch ermittelten FINKE et al. (1984) eine Abgangsrate von $46 \%$ nach dem ersten Wurf im Gegensatz zu der hier gefundenen von ca. $20 \%$ für DE- und DL-Sauen. LUCIA et al. (2000) untersuchten 7.973 Sauen nicht näher beschriebener Rassen und ermittelten als wichtigste Abgangsursache mit 33,6\% Reproduktionsstörungen, gefolgt von zu geringer Wurfgröße mit 20,6 \%. Die Unterschiede zu den hier ermittelten Prozentsätzen ergeben sich wahrscheinlich aus den unterschiedlichen Definitionen der Abgangsgründe, die im vorliegenden Datenmaterial nicht näher erläutert sind und gerade bei Reproduktions- und Zuchtleistungsproblemen sehr unterschiedliche Parameter beinhalten können. Der Prozentsatz an Sauen, die wegen zu hohen Alters aus dem Betrieb ausschieden, betrug jedoch wie bei den hier untersuchten Sauen etwa 8 \%. Bei LUCIA et al. (2000) wurden 14,9 \% nach dem ersten Wurf und $57 \%$ der Sauen vor dem 4 . Wurf gemerzt. Diese Werte sind nicht vollkommen vergleichbar mit den hier gefundenen von 19,7 bzw. 23,2 \% und 46,6 bzw. 56,8 \%, da LUCIA et al. (2000) die Prozentzahlen auf eine Gesamtzahl Sauen bezogen, in der auch Tiere beinhaltet waren, die vor dem ersten Wurf abgingen, wobei die hier untersuchten Sauen bereits wenigstens einen Wurf erbracht hatten.

Verteilung der Abgangsursachen auf die Abgangszeitpunkte

Die Selektion auf Zuchtleistung und ungestörtes Reproduktionsverhalten hat über alle Abgangswurfklassen einen gleichbleibend hohen Stellenwert bei allen Rassen, da diese Merkmale für eine wirtschaftliche Sauenhaltung unerlässlich sind. Der hohe Prozentsatz der Abgangsursache „Ausbleibende Trächtigkeit nach dem ersten Wurf“, lässt sich dadurch erklären, dass einmal aufgetretene Fruchtbarkeitsprobleme wie z.B. Umrauschen bzw. keine Rausche bereits am Anfang der Zuchtnutzung die Befürchtung 
aufkommen lassen, dass sie sich in den folgenden Zyklen der Sau wiederholen könnten und deshalb eine frühzeitige Merzung dieser Tiere sinnvoll erscheint. In einer früheren Untersuchung fanden DIJKHUIZEN et al. (1989) heraus, dass Sauen mit ausbleibender Trächtigkeit im Durchschnitt zu spät gemerzt werden, während Sauen mit nicht beobachtbarer Rausche jedoch häufig zu früh abgehen. Die Selektionsentscheidung wegen schlechter Mast- und Schlachtleistung der Ferkel findet hauptsächlich in dem Zeitraum zwischen dem 2. und 4. Wurf statt, da die Ergebnisse für dieses Merkmal erst mit einer gewissen Zeitverzögerung nach den ersten Würfen anfallen. Der Prozentsatz wegen Krankheit ausgeschiedener Tiere nimmt bei den höheren Abgangswurfklassen deutlich ab, da die hohe Nutzungsdauer dieser Sauen auf eine gute Fitness und ein ungestörtes Reproduktionsvermögen der Tiere schließen lässt, so dass sie eher wegen zu weit fortgeschrittenem Alter von der Zucht ausgeschlossen werden, wie man an der zeitlichen Entwicklung der Prozentsätze dieser Abgangsursache sieht.

Der prozentuale Anteil der Sauen, die wegen ausbleibender Trächtigkeit in den einzelnen Wurfklassen abgingen, war bei LUCIA et al. (2000) mit 19,3 \% wie bei den hier gefundenen Werten von 17,8 - 29,7 \% für den ersten Wurf am höchsten und nahm mit zunehmenden Alter der Sauen ab. Die auf Grund von schlechter Zuchtleistung abgegangenen Sauen erreichten bei LUCIA et al. (2000) zwischen dem 5. und 6. Wurf den höchsten Anteil mit 25,1 \%, während hier der größte Prozentsatz ( 5,8 bis 7,5 \%) bei den Abgängen nach dem 4. Wurf lag. Der größte Anteil Sauen mit der Abgangsursache „Hohes Alter“ lag bei LUCIA et al. (2000) nach dem 7.-8. Wurf (43,8 \%) und fiel danach auf $28 \%$ ab. Im Gegensatz dazu war der Prozentsatz für die Abgangsursache „Hohes Alter“ bei der vorliegenden Untersuchung für die Würfe 9 und größer am höchsten (43,4 - 46,9 \%), wobei der Prozentsatz Sauen, die nach dem 9. Wurf abgingen, hier auch um das 2 - 3-fache höher war als bei LUCIA et al. (2000). D'ALLAIRE et al. (1987) untersuchten die Abgangsursachen von 7.242 Sauen aus den USA ohne Rassenangabe und ermittelten ebenfalls den höchsten Prozentsatz an Sauen, die wegen Fruchtbarkeitsproblemen ausschieden, nach dem ersten Wurf (15,6 \%). Der Peak für die Abgangsursache „Geringe Zuchtleistung“ $(41,5)$ findet sich wie bei der vorliegenden Untersuchung um den 4 . Wurf, liegt aber deutlich höher als die hier ermittelten Werte von 5,8 bis 7,5 \%. Dies liegt an der unterschiedlichen Definition der Abgangsursachen, so fallen bei D’ALLAIRE et al. (1987) auch Geburtsgewicht und Zunahme der Ferkel mit unter diesen Punkt, während bei der vorliegenden Untersuchung diese Aspekte eher unter der Abgangsursache „Schlechte Mast- und Schlachtleistung“ zu finden sind. Die höchsten Prozentzahlen (36,9 \%) für die Abgangsursache „Hohes Alter“ finden sich bei D’ALLAIRE et al. (1987) für die Abgänge zwischen dem 7. und 9. Wurf, was nur ungefähr mit den eigenen Werten vergleichbar ist, da die Wurfklasseneinteilung geringfügig anders gestaltet wurde. Die Anteile der Abgangsursachen „Fruchtbarkeit“ und „Hohes Alter“ sind bei D’ALLAIRE et al. (1987) ebenfalls wie bei LUCIA et al. (2000) auf eine Gesamtzahl von Sauen bezogen, die Jungsauen ohne ersten Wurf mit einbezieht.

\section{Effekt der Abgangsursachen auf die Lebensleistung}

Die Höhe der LS-Mittelwerte der Lebensleistungsmerkmale „Lebens- und Nutzungsdauer“ sowie „Anzahl insgesamt lebend geborener bzw. aufgezogener Ferkel“ für die unterschiedlichen Abgangsursachen hängt unmittelbar mit dem Zeitpunkt der Selektionsentscheidung zusammen. So hatten Tiere mit altersbedingtem 
Abgang die höchsten LS-Mittelwerte, während Sauen, die wegen geringer Mast- und Schlachtleistung abgingen, die geringsten Werte erwarten lassen, da die Merzung wegen dieses Merkmals relativ früh stattfand. Für die Abgangsursache „Zuchtleistung und Fruchtbarkeit“ wurden ähnlich hohe LS-Mittelwerte geschätzt, da über alle Abgangswürfe hinweg der Einfluss dieser Abgangsursachen als Selektionsmerkmal recht hoch ist. So befanden sich die Schätzwerte für die Lebensleistung im Durchschnittsbereich der gesamten Sauen von ca. 4 Würfen.

LUCIA et al. (2000) ermittelten einen LS-Mittelwert für die Nutzungsdauer bei den Abgangsursachen „Krankheit“ bzw. „Zuchtleistung“ von 545 bzw. 680 Tagen was mit den hier gefundenen Werten von 549 bzw. 673 Tagen für DE- und DL-Sauen übereinstimmt. Auch die LS-Mittelwerte für die Anzahl insgesamt lebend geborener bzw. aufgezogener Ferkel von 38,6 bzw. 33,1 (42,2 bzw. 35,6 für die Zuchtleistung) deckt sich in etwa mit den hier ermittelten von ca. 37 bzw. ca. 35 (43 bzw. 39) Ferkeln bei den DE- und DL-Sauen. Bei den LS-Mittelwerten für die Nutzungsdauer von Sauen, die wegen hohen Alters abgingen, ergab sich jedoch eine Abweichung von ca. 3 Monaten zu den hier untersuchten DE-Sauen und sogar von knapp 6 Monaten zu den PISauen. Dies liegt daran, dass bei LUCIA et al. (2000) die Sauen mit der Abgangsursache hohes Alter deutlich früher abgingen als in der eigenen Untersuchung, so dass auch eine geringere Aufzuchtleistung im gesamten Leben zu erwarten ist. Dies wird durch die Werte von 79,8 bzw. 68,2 für die Anzahl insgesamt lebend geborener bzw. aufgezogener Ferkel bei LUCIA et al. (2000) im Vergleich zu ca. 82 bzw. 76 Ferkeln bei den DE- und DL-Tieren bestätigt. Der LS-Mittelwert für die Nutzungsdauer bei den wegen Fruchtbarkeitsproblemen abgegangenen Sauen betrug bei LUCIA et al. (2000) 441 Tage und für die Anzahl insgesamt lebend geborener bzw. aufgezogener Ferkel 32,7 bzw. 29,4. Dies liegt durchschnittlich 7,5 Monate und 7 bis 8 Ferkel unter den hier ermittelten Werten der DE- und DL-Sauen, was damit zusammenhängt, dass die von LUCIA et al. (2000) miteinbezogenen Sauen ohne Wurfleistung mit einem hohen Prozentsatz von ca. 36 \% in die Analyse eingingen, und dadurch die durchschnittliche Lebensleistung nach unten korrigiert wurde.

\section{Heritabilitätsschätzung}

Die Heritabilitätsschätzwerte für die Abgangsursache „Zuchtleistung“ waren mit $\mathrm{h}^{2}=$ 0,08 für die DE-Sauen deutlich höher als bei den anderen beiden Rassen, da diese Sauen hauptsächlich auf der Mutterseite eingesetzt werden und auf dieses Merkmal dort besonders viel Wert gelegt wird. Genau der gegenteilige Zustand war bei der Abgangsursache „Mast- und Schlachtleistung“ zu beobachten. Hier waren die Schätzwerte mit $h^{2}=0,06$ bzw. $h^{2}=0,09$ für die DL- bzw. PI-Sauen höher, da dieses Merkmal bei diesen beiden Rassen in der Selektion stark berücksichtigt wird. Für die Abgangsursache „Mangelnde Fruchtbarkeit“ konnten nur sehr geringe Heritabilitäten geschätzt werden, da die Ursachen für eine ausbleibende Trächtigkeit sehr vielseitig und unter anderem auch managementbedingt und somit tierunabhängig sein können. Auch bei der Abgangsursache „Krankheit“ wurden viele verschiedene Erkrankungen unter einem Punkt zusammengefasst. Die ähnlichen Werte von $\mathrm{h}^{2}=0,04$ bzw. $\mathrm{h}^{2}=$ 0,05 für die DE- bzw. die DL-Sauen können nur vermuten lassen, dass es sich hier hauptsächlich um ähnliche Organerkrankungen handelte. 


\section{Literatur}

DAGORN, J.; AUMAITRE, A.:

Sow culling: Reasons for and effect on productivity. Livest. Prod. Sci. 6 (1979), 167-177

D'ALLAIRE, S.; STEIN, T. E.; LEMAN, A. D.:

Culling patterns in selected Minnesota swine breeding herds. Can. J. Vet. Res. 51 (1987), 506-512

DIJKHUIZEN, A. A.; MORRIS, R. S.; MORROW, M.:

Economic optimization of culling strategies in swine breeding herd, using the "Porkshop" computer program. Prev. Vet. Med. 4 (1986), 341-352

DIJKHUIZEN, A. A.; KRABBENBORG, R. M. M.; HUIRNE, R. B. M.:

Sow replacement: A comparison of farmers' actual decisions and model recommendations. Livest. Prod. Sci. 23 (1989), 207-218

EIKMEIER, H.; MAYER, H.:

Untersuchungen über die Abgangsursachen von Zuchtsauen aus Herdbuchbetrieben. Tierärztl. Umschau 20 (1965), 282-284

FINKE, K.; SCHMITTEN F.; MUJICA, F.:

Einfluss von Rasse und Haltungsform auf die Reproduktionsleistung und Nutzungsdauer von Zuchtsauen. Züchtungskunde 56 (1984), 36-47

KROES, Y.; VAN MALE, J. P.:

Reproductive lifetime of sows in relation to economy of production. Livest. Prod. Sci. 6 (1979), 179183

LUCIA, T.; DIAL, G. D.; MARSH, W. E.:

Lifetime reproductive performance in female pigs having distinct reasons for removal. Livest. Prod. Sci. 63 (2000), 213-222.

PATTISON, H. D.; COOK, G. L.; MACKENZIE, S.:

A study of culling patterns in commercial pig breeding herds. Proc. Br. Soc. Anim. Prod. (1980), Harrogate, 462-463

STEIN, T. E.; DIJKHUIZEN, A.; D’ALLAIRE, S.; MORRIS R. S.:

Sow culling and mortality in commercial swine breeding herds. Prev. Vet. Med. 9 (1990), 85-94

TÖLLE, K.-H.; THOLEN, E.; TRAPPMANN ,W.; STORK , F.-J:

Möglichkeiten der Zuchtwertschätzung für Reproduktionsmerkmale beim Schwein am Beispiel eines Schweinezüchterverbandes. Züchtungskunde 70 (1998), 351-361

Eingegangen: 08.08.2003

Akzeptiert: 22.10.2003

Anschrift der Verfasser

MELANIE HEUSING, Dr. HENNING HAMANN, Prof. Dr. OTTMAR DISTL

Institut für Tierzucht und Vererbungsforschung der Tierärztlichen Hochschule Hannover

Bünteweg 17p

D-30559 Hannover

E-Mail: ottmar.distl@tiho-hannover.de 